\title{
D-Dimer Is a Predictive Factor of Cancer Therapeutics-Related Cardiac Dysfunction in Patients Treated With Cardiotoxic Chemotherapy
}

\begin{abstract}
Masayoshi Oikawa*, Daiki Yaegashi, Tetsuro Yokokawa, Tomofumi Misaka, Takamasa Sato, Takashi Kaneshiro, Atsushi Kobayashi, Akiomi Yoshihisa, Kazuhiko Nakazato, Takafumi Ishida and Yasuchika Takeishi
\end{abstract}

Department of Cardiovascular Medicine, Fukushima Medical University, Fukushima, Japan

Background: D-dimer is a sensitive biomarker for cancer-associated thrombosis, but little is known about its significance on cancer therapeutics-related cardiac dysfunction (CTRCD).

Edited by:

Dong Han,

People's Liberation Army General Hospital, China

Reviewed by:

Nicola Maurea,

G. Pascale National Cancer Institute

Foundation (IRCCS), Italy

Steven Philip Grover,

University of North Carolina at Chapel

Hill, United States

*Correspondence: Masayoshi Oikawa moikawa@fmu.ac.jp

Specialty section

This article was submitted to

Cardio-Oncology,

a section of the journal

Frontiers in Cardiovascular Medicine

Received: 02 November 2021

Accepted: 16 December 2021

Published: 21 January 2022

Citation:

Oikawa M, Yaegashi D, Yokokawa T, Misaka T, Sato T, Kaneshiro T, Kobayashi A, Yoshihisa A, Nakazato K,

Ishida T and Takeishi Y (2022)

$D$-Dimer Is a Predictive Factor of Cancer Therapeutics-Related Cardiac

Dysfunction in Patients Treated With

Cardiotoxic Chemotherapy.

Front. Cardiovasc. Med. 8:807754.

doi: 10.3389/fcvm.2021.807754
Methods: Consecutive 169 patients planned for cardiotoxic chemotherapy were enrolled and followed up for 12 months. All patients underwent echocardiography and blood test at baseline and at 3-, 6-, and 12 months.

Results: The patients were divided into two groups based on the level of D-dimer $(>1.65 \mu \mathrm{g} / \mathrm{ml}$ or $\leqq 1.65 \mu \mathrm{g} / \mathrm{ml})$ at baseline before chemotherapy: high D-dimer group $(n=37)$ and low D-dimer group $(n=132)$. Left ventricular ejection fraction (LVEF) decreased at 3- and 6 months after chemotherapy in high D-dimer group [baseline, $65.2 \%$ (62.8-71.4\%); 3 months, 62.9\% (59.0-67.7\%); 6 months, 63.1\% (60.0-67.1\%); 12 months, 63.3\% (58.8-66.0\%), $p=0.03$ ], but no change was observed in low D-dimer group. The occurrence of CTRCD within the 12-month follow-up period was higher in the high D-dimer group than in the low D-dimer group (16.2 vs. 4.5\%, $p=0.0146$ ). Multivariable logistic regression analysis revealed that high D-dimer level at baseline was an independent predictor of the development of CTRCD [odds ratio $3.93,95 \% \mathrm{Cl}$ (1.00-15.82), $p=0.047]$.

Conclusion: We should pay more attention to elevated D-dimer levels not only as a sign of cancer-associated thrombosis but also the future occurrence of CTRCD.

Keywords: cardio-oncology, D-dimer, cancer therapeutics-related cardiac dysfunction, heart failure, troponin I

\section{INTRODUCTION}

Recent advances in the diagnosis and treatment of cancers improve its prognosis. However, anticancer drugs, namely, anthracyclines, monoclonal antibodies, tyrosine kinase inhibitors, etc., induce cardiac dysfunction, resulting in poor prognosis in cancer survivors (1). Several cardiac biomarkers and echocardiographic parameters, such as troponins, myeloperoxidase, 
interleukin-1 $\beta$ (IL-1 $\beta$ ), Nucleotide-binding domain-like receptor family pyrin domain containing 3 , and reduced global longitudinal strain, are proposed to detect the early phase of cancer therapeutics-related cardiac dysfunction (CTRCD) and prompt cardioprotective treatment can improve cardiac function (2-7). Although those parameters are useful, careful monitoring is required for all patients to detect early signs of CTRCD. Thus, a novel biomarker that identifies high-risk patients before chemotherapy is desirable to perform effective clinical monitoring.

D-dimer is a sensitive biomarker for cancer-associated thrombosis, but accumulating evidence suggests that pretreatment $\mathrm{D}$-dimer can be used as a prognostic biomarker for patients with solid tumors (8). In cardiovascular fields, elevated $\mathrm{D}$-dimer is associated with not only thromboembolic events but also heart failure mortality in heart failure patients with reduced and preserved ejection fraction $(\mathrm{EF})(9,10)$.

Although $\mathrm{D}$-dimer is a promising biomarker in the cardiooncology field, little is known about the relationship between D-dimer and CTRCD. The present study aimed to evaluate the predictive impact of $\mathrm{D}$-dimer before chemotherapy on the development of CTRCD.

\section{METHODS}

\section{Study Subjects and Protocol}

We enrolled 202 consecutive cancer patients, planned for cardiotoxic chemotherapy, such as anthracyclines, human epidermal growth factor receptor 2 (HER2) inhibitors, tyrosine kinase inhibitors, and proteasome inhibitors, at Fukushima Medical University hospital from November 2016 to March 2019 (Figure 1). Patients were excluded if they died or were transferred to other hospitals within 12 months follow-up period $(n=33)$. The remaining 169 patients were divided into two groups based on the cut-off value of D-dimer, which was defined by the receiver operator characteristic curve analysis to detect the occurrence of CTRCD (Figure 2).

Hypertension was defined as a history of use of an antihypertensive drug or systolic blood pressure of $\geq 140 \mathrm{mmHg}$ and/or diastolic blood pressure $\geq 90 \mathrm{mmHg}$. Diabetes was defined as recent use of insulin treatment or hypoglycemic drug or hemoglobin A1c $\geq 6.5 \%$. Dyslipidemia was defined as a history of use of cholesterol-lowering drugs, or triglyceride was $\geq 150$ $\mathrm{mg} / \mathrm{dl}$, low-density lipoprotein cholesterol was $\geq 140 \mathrm{mg} / \mathrm{dl}$, and/or high-density lipoprotein cholesterol was $\leq 40 \mathrm{mg} / \mathrm{dl}$. A cumulative dose of anthracycline was expressed as a doxorubicin equivalent (1). HER2 inhibitors included trastuzumab and pertuzumab. Tyrosine kinase inhibitors included dabrafenib, trametinib, lenvatinib, sorafenib, dasatinib, bevacizumab, and pazopanib. Proteasome inhibitors included carfilzomib and bortezomib. Radiation therapy was defined as irradiation to the mediastinum and/or the heart field within the follow-up period. Transthoracic echocardiography and blood sampling test were performed at baseline as well as at 3, 6, and 12 months after the administration of cardiotoxic chemotherapy. All procedures used in this research were approved by the Ethical Committee of Fukushima Medical University.

\section{Echocardiography}

Transthoracic echocardiography was performed by a trained sonographer, and images were checked by another trained sonographer and an echo-cardiologist. We measured cardiac function using EPIQ 7G (Philips Healthtech, Best, The Netherlands). Left ventricular (LV) EF was calculated using the modified Simpson's method according to the guideline from the American Society of Echocardiography and the European Association of Cardiovascular Imaging (11). The LV mass was calculated using the following formula:

$$
\begin{aligned}
& \text { Left ventricular }(L V) \text { mass }=0.8 \\
& \times\left[1.04 \times\left\{\left(\begin{array}{l}
\text { LV diastolic diameter }+ \\
\text { interventricular septum wall } \\
\text { thicness }+L V \text { posterior wall thicness }
\end{array}\right)^{3}\right.\right. \\
& \left.\left.-(\text { LV diastolic diameter })^{3}\right\}\right]+0.6 \mathrm{~g}(11) .
\end{aligned}
$$

Cancer therapeutics-related cardiac dysfunction was defined as a decrease in EF by more than $10 \%$ points, to a value $<53 \%$ (12). The LV end-diastolic volume index, LV end-systolic volume index, LV mass index, and left atrial volume index were measured using the B-mode ultrasound.

\section{Blood Sampling}

High sensitivity cardiac troponin I (TnI) was measured using an assay based on Luminescent Oxygen Channeling Immunoassay technology and run on a Dimension EXL Integrated Chemistry System (Siemens Healthcare Diagnostics, Deerfield, IL, USA). B-type natriuretic peptide (BNP) levels were measured using a specific immunoradiometric assay (Shionoria BNP kit, Shionogi, Osaka, Japan). D-dimer was measured using a latex agglutination method (Lias Auto D-dimer Neo, Sysmex, Kobe, Japan).

\section{Statistical Analysis}

All statistical analyses were performed using Prism 9 (GraphPad Software, San Diego, CA, USA) or R software packages version 3.6.3 (R core team 2020, Vienna, Austria). We used the ShapiroWilk test to discriminate which variables were normally or not normally distributed. Normally distributed variables were shown as mean \pm SD. Non-normally distributed variables were indicated by a median with interquartile range. Category variables were shown in numbers and percentages. Student's $t$ test was used for variables following a normal distribution, the Mann-Whitney U-test was used for variables of the non-normal distribution, and the chi-square test was used for categorical variables. The time course of EF (baseline, 3-, 6-, and 12 months after the administration of anthracyclines) was evaluated using the Friedman test.

Logistic regression analysis was performed to identify the variables to predict the occurrence of CTRCD. We selected variables relating to the general condition and cardiac function, i.e., age, echocardiographic parameters, use of anthracyclines, BNP, hemoglobin, estimated glomerular filtration ratio, and the elevation of $\mathrm{D}$-dimer. The variables presenting $p<0.05$ in the 


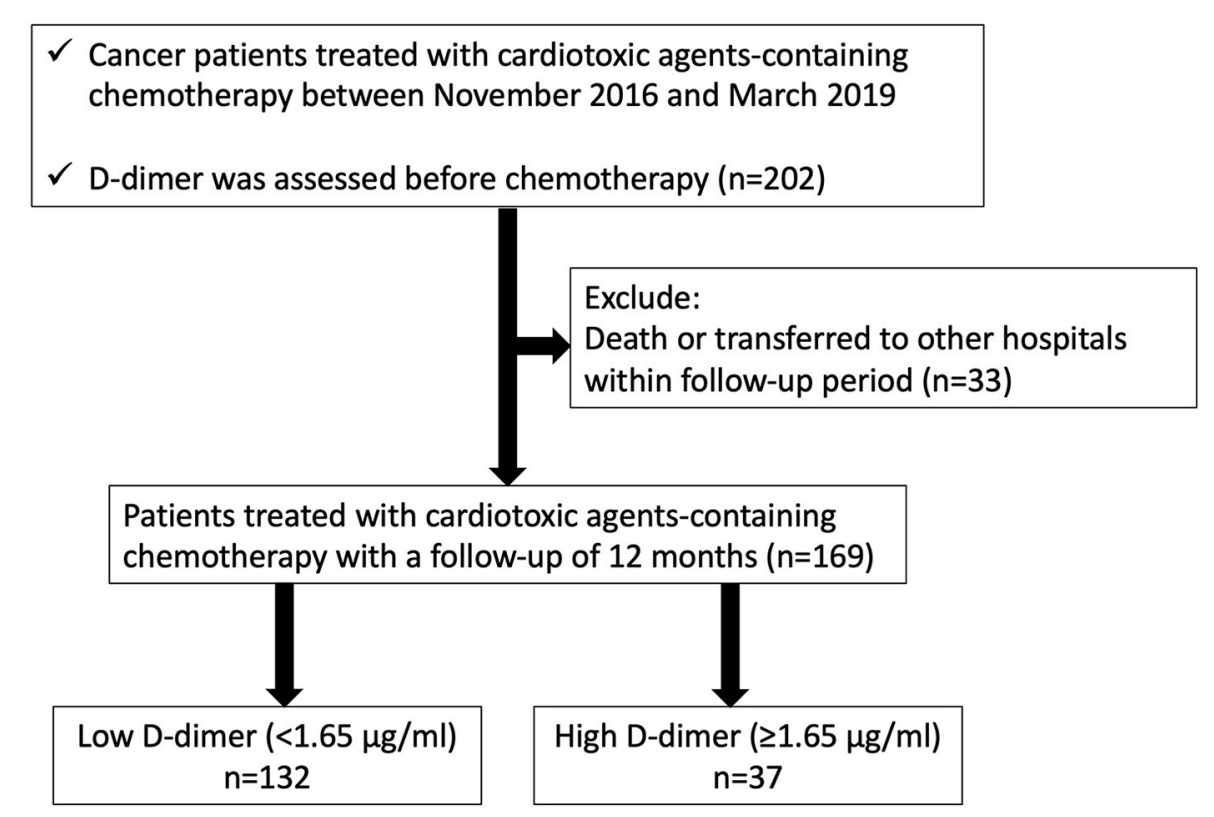

FIGURE 1 | Patient cohort selection.

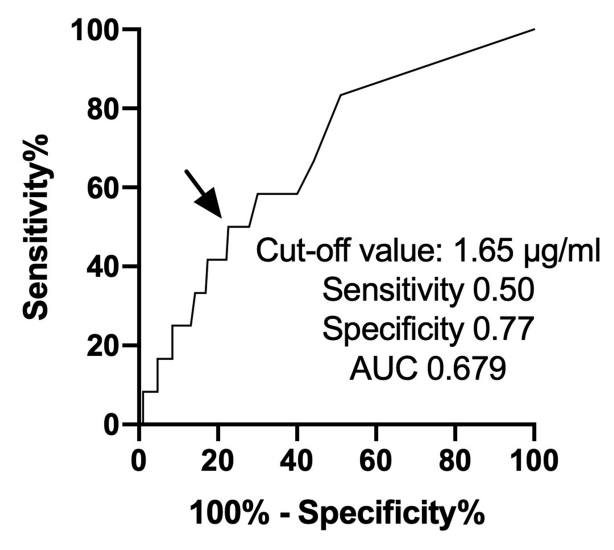

FIGURE 2 | ROC curve analysis of D-dimer predicting the occurrence of cancer therapeutics-related cardiac dysfunction. ROC, receiver operator characteristic curve.

univariable analysis were entered into the multivariable analysis. A receiver operating characteristic curve analysis was performed to determine the optimal cut-off value of the $\mathrm{D}$-dimer for predicting the occurrence of CTRCD. The $p$ of 0.05 or less was defined as significant.

\section{RESULTS}

First, we performed a receiver operating characteristic curve analysis to identify the threshold level of D-dimer to predict the occurrence of CTRCD (Figure 2). A total of 12 patients suffered from CTRCD within 12 months follow-up period. When we set the cut-off value of D-dimer at $1.65 \mu \mathrm{g} / \mathrm{ml}$, sensitivity, specificity, and area under the curve to predict CTRCD were 50.0\%, 80.3\%, and 0.661 , respectively. Then, we divided the patients into two groups based on the cut-off value. Table 1 shows patient characteristics at the baseline before chemotherapy. There were no statistical differences in age, sex, and the usage of angiotensinconverting enzyme inhibitors/angiotensin II receptor blockers and $\beta$-blockers. The high $\mathrm{D}$-dimer group included a lower rate of breast cancer (35 vs. 67\%, $p=0.0005$ ), a higher rate of ovarian/uterine cancer ( 19 vs. $6 \%, p=0.0151$ ), and a higher rate of leukemia ( 16 vs. $4 \%, p=0.0068$ ) than low D-dimer group. Echocardiographic data demonstrated that EF was slightly higher in the high $\mathrm{D}$-dimer group (67 \pm 5 vs. $64 \pm 5 \%, p=$ $0.0019)$. In laboratory data, the high $\mathrm{D}$-dimer group showed lower hemoglobin values and higher BNP values.

Time-dependent changes in EF are displayed in Figure 3. Low D-dimer group showed no changes in EF within the followup period, but EF was decreased at 3- and 6 months after chemotherapy in high D-dimer group [baseline, 65.2\% (62.8$71.4 \%)$; 3 months, $62.9 \%$ (59.0-67.7\%); 6 months, $63.1 \%$ (60.0$67.1 \%$ ); 12 months, 63.3\% (58.8-66.0\%), $p=0.03$, Figures 3A,B]. The reduction of EF from baseline was larger in high $\mathrm{D}$-dimer group than in low $\mathrm{D}$-dimer group ( 3 months: $-4.0 \pm 7.1$ vs. -0.5 $\pm 5.3, p=0.0015 ; 6$ months: $-4.8 \pm 8.0$ vs. $-0.2 \pm 6.2, p=$ $0.0004 ; 12$ months: $-4.5 \pm 7.3$ vs. $-0.4 \pm 6.6 \%, p=0.0024)$.

The occurrence of CTRCD during the 12-month followup period was higher in the high $\mathrm{D}$-dimer group than in the low D-dimer group (16.2 vs. $4.5 \%, p=0.0146)$. Multivariable logistic regression analysis revealed that $\mathrm{LV}$ end-diastolic volume index [odds ratio $0.95,95 \% \mathrm{CI}(0.91-0.99), p=0.0122$ ] and high D-dimer levels [odds ratio 3.93, 95\% CI (1.00-15.82), $p=$ 0.0469 ] before chemotherapy were independent predictors of the development of CTRCD (Table 2). 
TABLE 1 | Baseline clinical characteristics of patients with elevated or non-elevated D-dimer.

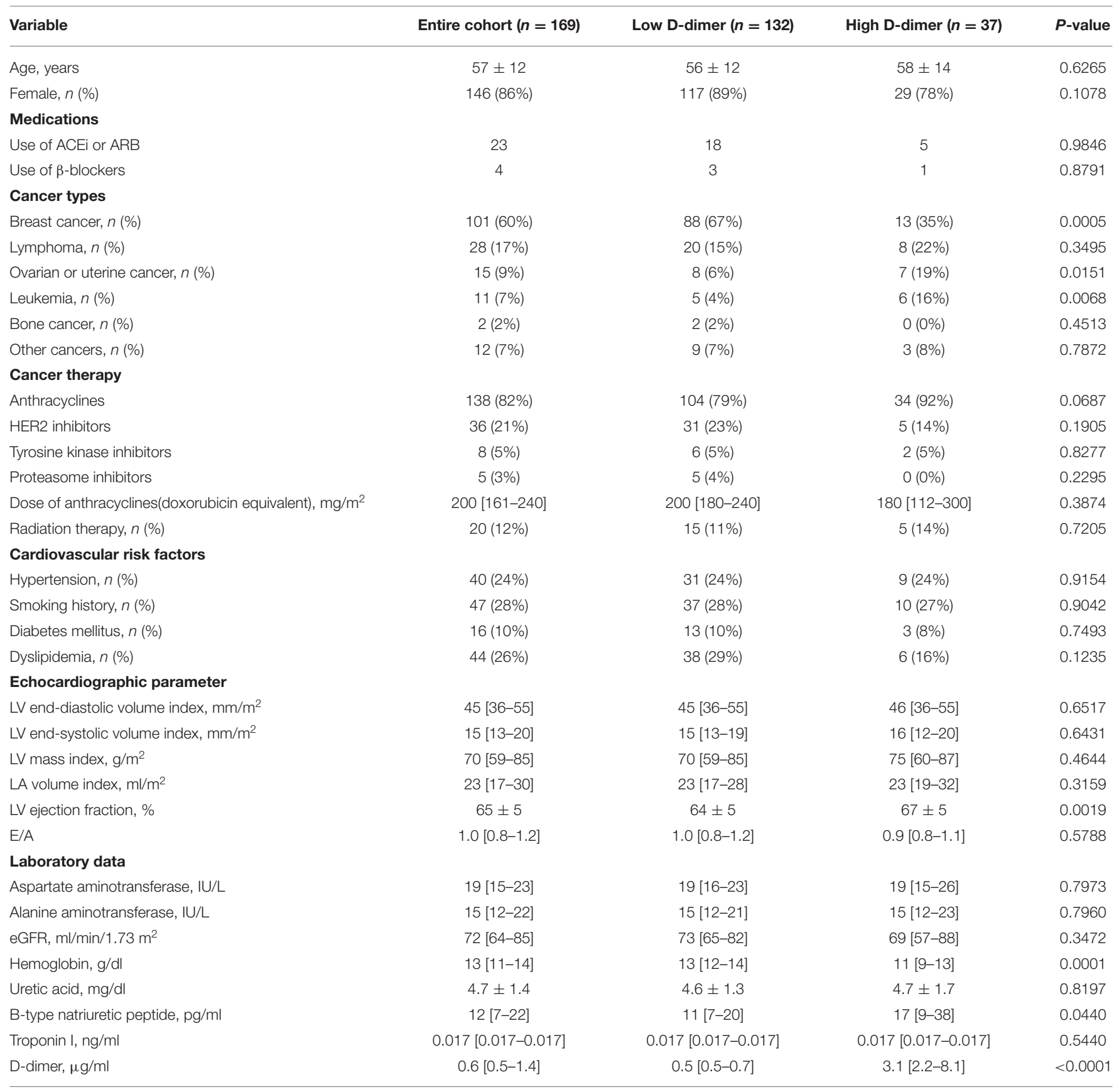

ACEi, angiotensin converting enzyme inhibitor; ARB, angiotensin II receptor blocker; HER2, human epidermal growth factor receptor 2; LV, left ventricular; E/A, early to late diastolic transmitral flow velocity; eGFR, estimated glomerular filtration ratio.

\section{DISCUSSION}

In the present study, we revealed the predictive features of D-dimer in patients treated with cardiotoxic agents. First, the threshold level of D-dimer was $1.65 \mu \mathrm{g} / \mathrm{ml}$ to predict the development of CTRCD. Second, EF was decreased time-dependently in high D-dimer patients. Third, the occurrence of CTRCD was significantly higher in high D-dimer patients.
D-dimer is a pivotal biomarker of hypercoagulability and thrombosis. Fibrin-bound plasmin degrades the fibrin network into soluble fragments D-dimers and $\mathrm{E}$ fragments, thus increased levels of D-dimer represent a global activation of coagulation and fibrinolysis (13). Cancers produce hypercoagulable and prothrombotic situations by secreting several prothromboembolic factors, such as mucins, cysteine protease, and tissue factors (14). Therefore, thrombi are easily generated in patients with cancer, and thromboembolism is the second 
A

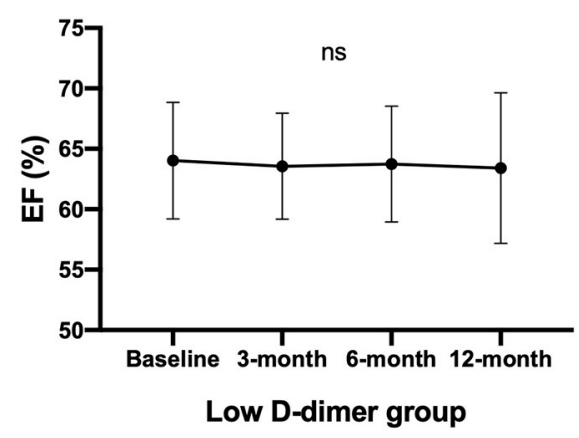

B

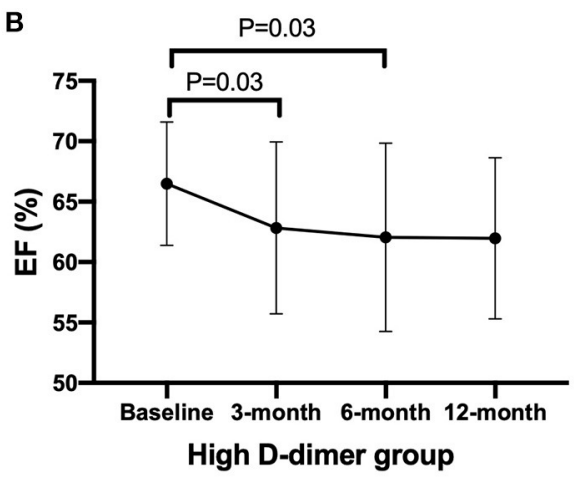

FIGURE 3 | Time-dependent changes in EF after chemotherapy. Data are expressed in mean with SD. Statistics is performed using Friedman's test with Dunn's multiple comparisons test. EF, ejection fraction. (A) Changes in EF in the low D-dimer group. (B) Changes in EF in the high D-dimer group. EF, ejection fraction.

TABLE 2 | Parameters associated with the occurrence of CTRCD.

\begin{tabular}{|c|c|c|c|c|}
\hline & \multicolumn{2}{|c|}{ Univariate } & \multicolumn{2}{|c|}{ Multivariate } \\
\hline & OR $(95 \% \mathrm{Cl})$ & $P$-value & OR $(95 \% \mathrm{Cl})$ & $P$-value \\
\hline Age, per 1 year increase & $1.01(0.96-1.05)$ & 0.8470 & & \\
\hline Male & $1.79(0.32-33.57)$ & 0.5852 & & \\
\hline Use of anthracyclines & $1.18(0.29-7.94)$ & 0.8353 & & \\
\hline BNP, per $1 \mathrm{pg} / \mathrm{ml}$ increase & $0.99(0.98-1.02)$ & 0.4239 & & \\
\hline LV ejectioin fraction, per $1 \%$ increase & $1.07(0.95-1.22)$ & 0.2591 & & \\
\hline LV end-diastolic volume index, per $1 \mathrm{ml} / \mathrm{m}^{2}$ increase & $0.95(0.91-0.99)$ & 0.0099 & $0.95(0.91-0.99)$ & 0.0122 \\
\hline E/A, per 1 increase & $0.24(0.05-1.39)$ & 0.0934 & & \\
\hline Left atrial volume index, per $1 \mathrm{ml} / \mathrm{m}^{2}$ increase & $0.99(0.94-1.05)$ & 0.7157 & & \\
\hline Hemoglobin, per $1 \mathrm{~g} / \mathrm{dl}$ increase & $1.13(0.85-1.45)$ & 0.3539 & & \\
\hline Estimated GFR, per $1 \mathrm{ml} / \mathrm{min} / 1.73 \mathrm{~m}^{2}$ & $0.98(0.95-1.02)$ & 0.2989 & & \\
\hline Elevated D-dimer (1.65 mg/dl) & $4.07(1.20-13.84)$ & 0.0218 & $3.93(1.00-15.82)$ & 0.0469 \\
\hline
\end{tabular}

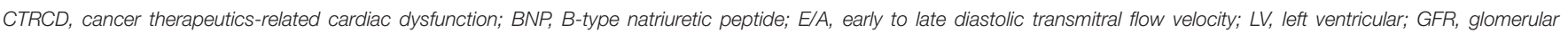
filtration ratio.

leading cause of cancer-related morbidity and mortality $(15,16)$. Although D-dimer is an established and widely used biomarker for the screening of thrombus formation in patients with cancer, prognostic features of $\mathrm{D}$-dimer have become clinically overt recently. The link between $\mathrm{D}$-dimer and cancer progression is reported in several papers $(17,18)$, and higher levels of D-dimer are associated with poor prognosis in cancer patients (18). Although the precise mechanisms are still complex and uncovered, the pro-coagulable state may produce a suitable milieu for cancer progression by recruitment of pro-metastatic leukocytes, adhesion to the endothelium, transendothelial migration, and restriction in natural killer cell-mediated clearance of micrometastasis $(19,20)$. Accumulating evidence showed that abnormal inflammation and oxidative stress are key factors to the development of heart failure, and these also play important roles in cancer progression and thrombus formation (21-25). For example, IL-1 $\beta$, a representative inflammatory cytokine, induces cardiac dysfunction and thrombus formation (26). IL-1 $\beta$ activates myddosome complex, such as nuclear factor $\kappa \mathrm{B}$, myeloid differentiation factor 88 , cryopyrin, and p38-MAPK, in cardiomyocytes, leading to dysregulates metabolism in the sarcoplasmic reticulum, calcium homeostasis, and myocardial apoptosis and necrosis (7). In addition, IL-1 $\beta$ increased pro-coagulant state through activating tissue factor-dependent mechanisms in endothelial cells (27). Gomes et al. reported that blockade of IL-1 receptor abolished the neutrophil extracellular traps-dependent prothrombotic state and attenuated cancer-associated thrombosis in murine breast cancer model (25). Considering the fact that inflammation is a major contributor to cardiac dysfunction and thrombus formation, cancer patients with high D-dimer may be predisposed to cardiac dysfunction due to a chronic inflammatory state. Cardiotoxic chemotherapeutic agents are crucial and indispensable to performing cancer treatment. Anthracyclines induce pro-inflammatory responses by increasing tumor necrosis factor $\alpha$ (TNF- $\alpha$ ), IL-1 $\beta$, and IL-6, leading to tumor cell death (28). Not only anthracyclines but also targeted chemotherapy, such as trastuzumab and bevacizumab, increased inflammatory cytokines after the treatment $(29,30)$. In the present study, the patients with a high $\mathrm{D}$-dimer group may already have been exposed to an inflammatory state before chemotherapy and were vulnerable to additional inflammatory 
stress by cardiotoxic agents, resulting in the development of CTRCD. To elucidate the precise mechanisms was beyond this study, but the importance of $\mathrm{D}$-dimer should be noted in the cardio-oncology field. Intervention with Pravastatin in Ischemic Disease (LIPID) study revealed that elevated D-dimer levels predict long-term risk of arterial and venous events, cardiovascular disease mortality, in addition to that, increased cancer incidence and mortality rate (31). To the best of our knowledge, this is the first report assessing the relationship between D-dimer levels and the development of CTRCD. The importance of D-dimer should be taken into account when managing patients with cancer who are treated with cardiotoxic chemotherapy.

\section{LIMITATION}

This study has several limitations. First, this study was performed using a relatively small number of patients and a short followup period by a single center. Slight differences in EF at baseline may be due to the small sample size of the high $\mathrm{D}$-dimer group. Second, although not statistically significant, a higher proportion of patients in the high $\mathrm{D}$-dimer group received anthracycline-containing chemotherapies. This might affect the results in the reduction in EF in the high D-dimer group. Longer follow-up and larger population data were needed to confirm the importance of D-dimer to the development of CTRCD and cardiovascular prognosis. Third, D-dimer has modest sensitivity and specificity to predict CTRCD in the present study. The mechanisms by which CTRCD development must be complicated, thereby predicting CTRCD by a single biomarker is still challenging. D-dimer is frequently analyzed in daily clinical practice to detect cancer-associated thrombosis. Therefore, we think D-dimer is easy and useful for predicting both CTRCD and thrombus formation.

\section{REFERENCES}

1. Zamorano JL, Lancellotti P, Munoz DR, Aboyans V, Asteggiano R, Galderisi M, et al. 2016 ESC position paper on cancer treatments and cardiovascular toxicity developed under the auspices of the ESC committee for practice guidelines: the task force for cancer treatments and cardiovascular toxicity of the European society of cardiology (ESC). Eur Heart J. (2016) 37:2768801. doi: 10.1093/eurheartj/ehw211

2. Oikawa M, Yoshihisa A, Yokokawa T, Misaka T, Yaegashi D, Miyata $\mathrm{M}$, et al. Cardiac troponin I predicts elevated B-type natriuretic peptide in patients treated with anthracycline-containing chemotherapy. Oncology. (2020) 98:653-60. doi: 10.1159/000507585

3. Ky B, Putt M, Sawaya H, French B, Januzzi JL Jr, Sebag IA, et al. Early increases in multiple biomarkers predict subsequent cardiotoxicity in patients with breast cancer treated with doxorubicin, taxanes, and trastuzumab. J Am Coll Cardiol. (2014) 63:809-16. doi: 10.1016/j.jacc.2013.10.061

4. Thavendiranathan P, Negishi T, Somerset E, Negishi K, Penicka M, Lemieux J, et al. Strain-guided management of potentially cardiotoxic cancer therapy. J Am Coll Cardiol. (2021) 77:392-401. doi: 10.1016/j.jacc.2020. 11.020

5. Cardinale D, Colombo A, Bacchiani G, Tedeschi I, Meroni CA, Veglia F, et al. Early detection of anthracycline cardiotoxicity and improvement with heart failure therapy. Circulation. (2015) 131:1981-8. doi: 10.1161/CIRCULATIONAHA.114.013777

\section{CONCLUSION}

Elevated D-dimer is a pivotal biomarker to predict CTRCD. $\mathrm{D}$-dimer should be taken into account when managing cancer patients treated with cardiotoxic chemotherapy.

\section{DATA AVAILABILITY STATEMENT}

The raw data supporting the conclusions of this article will be made available by the authors, without undue reservation.

\section{ETHICS STATEMENT}

The studies involving human participants were reviewed and approved by Ethical Committee of Fukushima Medical University Hospital. The patients/participants provided their written informed consent to participate in this study.

\section{AUTHOR CONTRIBUTIONS}

MO created the study design, analyzed the data, and drafted the manuscript. DY created the study design and analyzed the data. TM, TS, TK, and AK acquired the data. AY, KN, TI, and YT interpreted the data and revised the manuscript. All authors contributed to the conception, design, critical revision, and final approval of this manuscript.

\section{FUNDING}

JSPS KAKENHI (Grant Number JP20K08493).

\section{ACKNOWLEDGMENTS}

The authors thank Ms. H. Kobayashi for data management.

6. Quagliariello V, De Laurentiis M, Cocco S, Rea G, Bonelli A, Caronna A, et al. NLRP3 as putative marker of ipilimumab-induced cardiotoxicity in the presence of hyperglycemia in estrogen-responsive and triple-negative breast cancer cells. Int J Mol Sci. (2020) 21:7802. doi: 10.3390/ijms21207802

7. Quagliariello V, Paccone A, Iovine M, Cavalcanti E, Berretta M, Maurea C, et al. Interleukin-1 blocking agents as promising strategy for prevention of anticancer drug-induced cardiotoxicities: possible implications in cancer patients with COVID-19. Eur Rev Med Pharmacol Sci. (2021) 25:6797812. doi: 10.26355/eurrev_202111_27124

8. Li W, Tang Y, Song Y, Chen SH, Sisliyan N, Ni M, et al. Prognostic role of pretreatment plasma D-dimer in patients with solid tumors: a systematic review and meta-analysis. Cell Physiol Biochem. (2018) 45:166376. doi: $10.1159 / 000487734$

9. Zhang X, Wang S, Sun L, Fang S, Yu B. Prognostic value of Ddimer in acute myocardial infarction complicated by heart failure with preserved ejection fraction. ESC Heart Fail. (2020) 7:4118-25. doi: 10.1002/ ehf2.13027

10. de Boer RA, Nayor M. deFilippi CR, Enserro D, Bhambhani V, Kizer JR, et al. Association of cardiovascular biomarkers with incident heart failure with preserved and reduced ejection fraction. JAMA Cardiol. (2018) 3:21524. doi: 10.1001/jamacardio.2017.4987

11. Lang RM, Badano LP, Mor-Avi V, Afilalo J, Armstrong A, Ernande L, et al. Recommendations for cardiac chamber quantification by echocardiography in adults: an update from the American Society of Echocardiography and 
the European Association of Cardiovascular Imaging. J Am Soc Echocardiogr. (2015) 28:1-39. doi: 10.1016/j.echo.2014.10.003

12. Plana JC, Galderisi M, Barac A, Ewer MS, Ky B, Scherrer-Crosbie M, et al. Expert consensus for multimodality imaging evaluation of adult patients during and after cancer therapy: a report from the American Society of Echocardiography and the European Association of Cardiovascular Imaging. J Am Soc Echocardiogr. (2014) 27:911-39. doi: 10.1016/j.echo.2014.07.012

13. Kyrle PA, Eichinger S. D-dimer for long-term risk prediction in patients after acute coronary syndrome. Jack of all trades, or master of none? Circulation. (2018) 138:724-6. doi: 10.1161/CIRCULATIONAHA.118.033670

14. Varki A. Trousseau's syndrome: multiple definitions and multiple mechanisms. Blood. (2007) 110:1723-9. doi: 10.1182/blood-2006-10-053736

15. Prandoni P, Falanga A, Piccioli A. Cancer and venous thromboembolism. Lancet Oncol. (2005) 6:401-10. doi: 10.1016/S1470-2045(05)70207-2

16. Tuzovic M, Herrmann J, Iliescu C, Marmagkiolis K, Ziaeian B, Yang EH. Arterial thrombosis in patients with cancer. Curr Treat Options Cardiovasc Med. (2018) 20:40. doi: 10.1007/s11936-018-0635-x

17. Han D. ó Hartaigh B, Lee JH, Cho IJ, Shim CY, Chang HJ, et al. Impact of D-dimer for prediction of incident occult cancer in patients with unprovoked venous thromboembolism. PLoS ONE. (2016) 11:e0153514. doi: 10.1371/journal.pone.0153514

18. Ay C, Dunkler D, Pirker R, Thaler J, Quehenberger P, Wagner O, et al. High D-dimer levels are associated with poor prognosis in cancer patients. Haematologica. (2012) 97:1158-64. doi: 10.3324/haematol.2011.05 4718

19. Lucotti S, Muschel RJ. Platelets and Metastasis: New implications of an old interplay. Front Oncol. (2020) 10:1350. doi: 10.3389/fonc.2020.01350

20. Palumbo JS, Talmage KE, Massari JV, La Jeunesse CM, Flick MJ, Kombrinck KW, et al. Tumor cell-associated tissue factor and circulating hemostatic factors cooperate to increase metastatic potential through natural killer cell-dependent and-independent mechanisms. Blood. (2007) 110:13341. doi: 10.1182/blood-2007-01-065995

21. Bertero E, Canepa M, Maack C, Ameri P. Linking heart failure to cancer: Background evidence and research perspectives. Circulation. (2018) 138:73542. doi: 10.1161/CIRCULATIONAHA.118.033603

22. Koene RJ, Prizment AE, Blaes A, Konety SH. Shared risk factors in cardiovascular disease and cancer. Circulation. (2016) 133:110414. doi: 10.1161/CIRCULATIONAHA.115.020406

23. Barrera G. Oxidative stress and lipid peroxidation products in cancer progression and therapy. ISRN Oncol. (2012) 2012:137289. doi: 10.5402/2012/137289

24. Jang JH, Kim DH, Surh YJ. Dynamic roles of inflammasomes in inflammatory tumor microenvironment. NPJ Precis Oncol. (2021) 5:18. doi: 10.1038/s41698-021-00154-7

25. Gomes T, Várady CBS, Lourenço AL, Mizurini DM, Rondon AMR, Leal AC, et al. IL-1 $\beta$ blockade attenuates thrombosis in a neutrophil extracellular trapdependent breast cancer model. Front Immunol. (2019) 10:2088. doi: 10.3389/ fimmu. 2019.02088
26. Bevilacqua MP, Pober JS, Majeau GR, Cotran RS, Gimbrone MA Jr. Interleukin 1 (IL-1) induces biosynthesis and cell surface expression of procoagulant activity in human vascular endothelial cells. J Exp Med. (1984) 160:618-23. doi: 10.1084/ jem.160.2.618

27. Puhlmann M, Weinreich DM, Farma JM, Carroll NM, Turner EM, Alexander HR. Jr. Interleukin-1beta induced vascular permeability is dependent on induction of endothelial tissue factor (TF) activity. J Transl Med. (2005) 3:37. doi: 10.1186/1479-5876-3-37

28. Tocchetti CG, Ameri P, de Boer RA, D’Alessandra Y, Russo M, Sorriento D, et al. Cardiac dysfunction in cancer patients: beyond direct cardiomyocyte damage of anticancer drugs: novel cardio-oncology insights from the joint 2019 meeting of the ESC Working Groups of Myocardial Function and Cellular Biology of the Heart. Cardiovasc Res. (2020) 116:182034. doi: $10.1093 / \mathrm{cvr} / \mathrm{cvaa} 222$

29. Dhesy-Thind S, Kumar V, Snider-McNair A, Shortt C, Mukherjee $\mathrm{SD}$, Ellis $\mathrm{P}$, et al. Cardiac and inflammation biomarker profile after initiation of adjuvant trastuzumab therapy. Clin Chem. (2013) 59:327-9. doi: 10.1373/clinchem.2012.192419

30. El-Hajjar L, Jalaleddine N, Shaito A, Zibara K, Kazan JM, El-Saghir $\mathrm{J}$, et al. Bevacizumab induces inflammation in MDA-MB-231 breast cancer cell line and in a mouse model. Cell Signal. (2019) 53:40012. doi: 10.1016/j.cellsig.2018.11.007

31. Simes J, Robledo KP, White HD, Espinoza D, Stewart RA, Sullivan $\mathrm{DR}$, et al. D-dimer predicts long-term cause-specific mortality, cardiovascular events, and cancer in patients with stable coronary heart disease: LIPID study. Circulation. (2018) 138:712-23. doi: 10.1161/ CIRCULATIONAHA.117.029901

Conflict of Interest: The authors declare that the research was conducted in the absence of any commercial or financial relationships that could be construed as a potential conflict of interest.

Publisher's Note: All claims expressed in this article are solely those of the authors and do not necessarily represent those of their affiliated organizations, or those of the publisher, the editors and the reviewers. Any product that may be evaluated in this article, or claim that may be made by its manufacturer, is not guaranteed or endorsed by the publisher.

Copyright (c) 2022 Oikawa, Yaegashi, Yokokawa, Misaka, Sato, Kaneshiro, Kobayashi, Yoshihisa, Nakazato, Ishida and Takeishi. This is an open-access article distributed under the terms of the Creative Commons Attribution License (CC BY). The use, distribution or reproduction in other forums is permitted, provided the original author(s) and the copyright owner(s) are credited and that the original publication in this journal is cited, in accordance with accepted academic practice. No use, distribution or reproduction is permitted which does not comply with these terms. 